\title{
SOLAR MASS EJECTIONS AND CORONAL HOLES
}

\author{
ARVIND BHATNAGAR \\ Udaipur Solar Observatory \\ (Physical Research Laboratory, Govt. of India, Dept. of Space) \\ 11, Vidya Marg, Udaipur 313001, India.
}

September 7, 1996

\begin{abstract}
In this paper we present observations of two types of solar mass ejections, which seem to be associated with the location of coronal holes. In the first type, a filament eruption was observed near a coronal hole, which gave rise to a strong interplanetary scintillations. as detected by IPS observations. In the second type, several large scale soft $\mathrm{X}$-ray 'blow-outs' were observed in the YOHKOH SXT X-ray movies, in all the cases they erupted from or near the boundary of coronal holes and over the magnetic neutral line. It is proposed that the open magnetic field configuration of the coronal hole provides, the necessary field structure for reconnection to take place, which in turn is responsible for filament eruption, from relatively lower heights. While, in the case of X-ray 'blow-outs', the reconnection takes place at a greater height, resulting in high temperature soft X-ray emission visible as X-ray 'blow-outs'.
\end{abstract}

Key words: Coronal hole-interplanetary shock-eruptive prominence-solar flare-solar soft X-rays-YOHKOH.

\section{Introduction}

The most energetic disturbances in the heliosphere near the Earth are due to interplanetary shocks. These shocks are accompanied by $0.1-0.2 \mathrm{AU}$ thick shells of increased density of energetic particles, followed by out flow of enhanced solar wind (Borrini et al., 1982). When such disturbances encounter the Earth, they interact with it's magnetosphere, causing geomagnetic storms and other solar - terrestrial effects. Obviously, the Sun in some way is responsible for interplanetary shocks, however, it is still unclear how and where they are generated. The most energetic transient energy release on the Sun is known to be associated with solar flares. Therefore, one obvious assumption would be that interplanetary shocks are perhaps generated due to flares. However, Joselyn and McIntosh(1981) and more recently Gosling (1993) have shown that strong shocks can occur in complete absence of flares. They have suggested that shocks may be related to disappearing filaments and prominences. In early 70's, the Skylab space mission revealed two important solar phenomena - corotating coronal holes and the coronal mass ejections (CME) detected by Thomson scattering of white light near the Sun. It is well established that geomagnetic activity is related to the appearance of coronal holes on the Sun. Hewish et al.,(1985) and Hewish and Bravo(1986) have shown that mid-latitude coronal holes lay near the sources of erupting 
short lived stream of energetic particles. Further, they showed that on many occasions no major flare or disappearing filament activity was seen any where on the disk, within the time interval of the observed shock. On the basis of these observations they concluded that perhaps some coronal hole activity was responsible for erupting streams and interplanetary shocks. Sheeley et al.,(1984) have shown that coronal mass ejections (CMEs) are closely associated with interplanetary shocks. However, the machanism of generation of CMEs is not yet well understood.

Wagner(1984) has shown that 10-17\% of CMEs are associated with flares, $30-40 \%$ with erupting prominences and $30-48 \%$ are unrelated to any optical observed phenomena on the solar surface. Evidently, some of the CMEs must be generated by some unknown fundamental process, unrelated to flares and erupting promenences. From interplanetary scintillation (IPS), ground based $\mathrm{H} \alpha$ and soft X-ray observations from YOHKOH SXT, we present in this paper a possible relation between coronal hole, solar mass ejection and X-ray 'blow-out'.

\section{Relation between solar mass ejection, coronal hole and interplanetary disturbance (IPD)}

It has been well established that a good fraction of observed CMSs and IPDs are associated with eruptive prominences, filament disappearance and flares. A class X1/2B Tenflare occured on October 30,1992 at 08:16 UT in NOAA region $7321(\mathrm{~S} 25, \mathrm{~L}=71)$. The $\mathrm{H} \alpha$ observations made at the Udaipur Solar Observatory revealed that a filament in the active region erupted before or around the flare maximum. Following this event, Janardhan et al.,(1994) observed on 31 October 1992, a strong IPD through the Ooty IPS radio telescope. A low latitude coronal hole lay about 10 degrees east of the active region, as shown in Figure 1. Using the back projection technique, it was confirmed that the observed IPD originated from or near the same active region NOAA 7321, and triggered by the flare and the mass ejection observed near the coronal hole. Thus, it appears that all the three phenomena - flare, erupting filament and the coronal hole were associated with the observed IPD.

We propose that this IPD event, occurred due to the reconnection of open field lines of the coronal hole, with the emerging flux from the active region. This process led to the flare and mass ejection. The ejected filament material gave rise to enhanced density and high speed in the interplaneytary medium, resulting in the observed IPD. In Figure 2, is given a possible scenario of the magnetic field configuration near the coronal hole and the active region. 


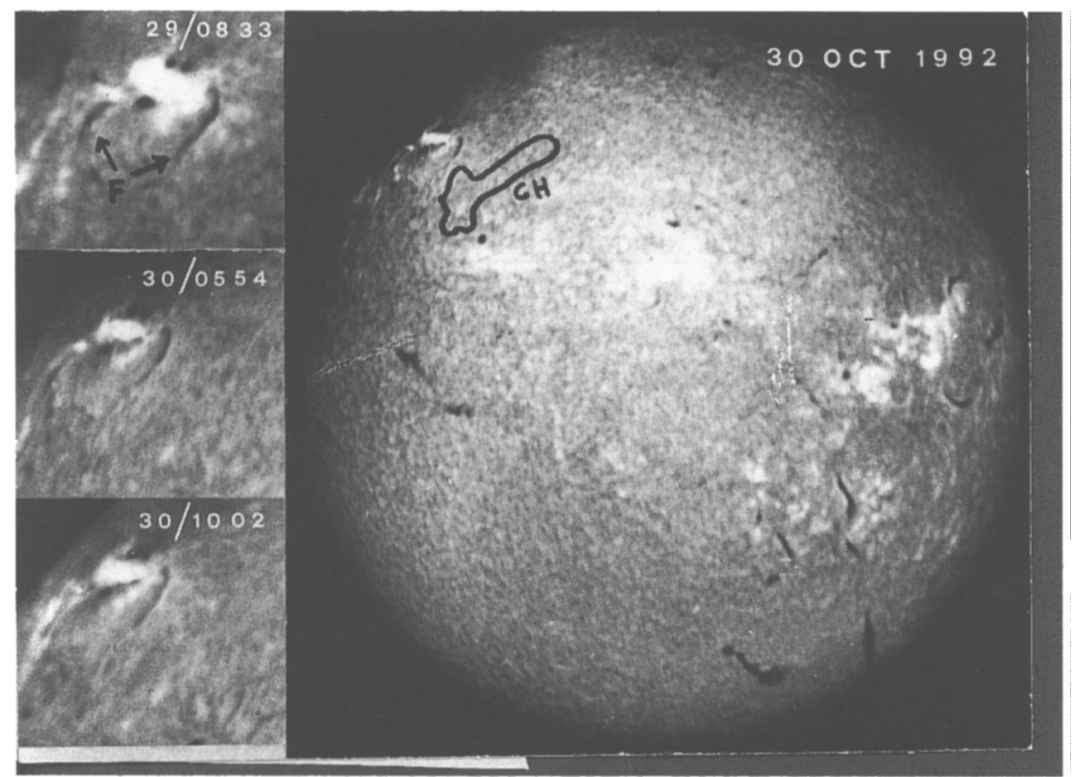

Fig. 1. H $\alpha$ fitergram taken on 29 and 30 Oct 1992, showing the filament disappearence and location of coronal hole.

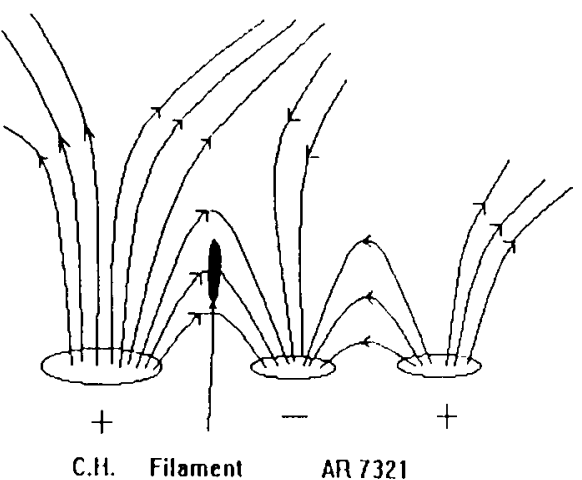

[1]

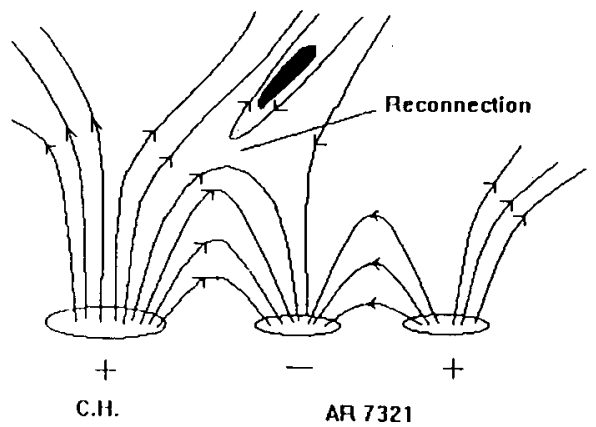

(III)

Fig. 2. A proposed scenario of magnetic field configuration near active region, filament and coronal hole. 


\section{RELATION BETWEEN CORONAL HOLES AND SOFT X-RAY 'BLOW-OUT'}

Recent soft X-ray observations made by YOHKOH SXT has revealed wealth of solar phenomena associated with both flare and non-flare activities. For this discussion, we consider one of the interesting non-flare phenomenon of ejection of 'Soft X-ray CMEs', as defined by Hudson (1994). From a survey of SXT routine movies, Klimchuk et al., (1994) have identified many soft X-ray ejections and found a mean projected speed of $48 \mathrm{~km} / \mathrm{s}$. Besides these, SXT has observed more rapid transients associated with active regions (Watanabe et al., 1992) and polar latitudes (Hiei et al., 1993). Hudson (1994) reported that at least, some of these ejections (if not all) correspond to coronal mass ejections, as observed by coronagraphs. From SXT data, Khan et al., (1994), McAllister et al., (1992), Kahler(1992) and Tsuneta et al., (1992) have shown that huge soft X-ray structures develop both in active region latitudes (but out side active regions) and in polar crown filament zone.

We have examined YOHKOH SXT movies to look for Soft X-ray CMEs and a possible relation with coronal holes and other solar surface activity. We found that number of Soft X-ray ejections occurred in quiet regions in the form of 'blow-outs', extending in some cases even more than half the solar radius ( 3 to $400,000 \mathrm{~km}$ ). From the visual appearance of this phenomenon, it may be appropriate to call it as 'X-ray BLOW-OUT'(XBO), similar to streamer blow out, a term used in coronal observations. From the YOHKOH SXT movies, we have selected 15 cases of distinct XBOs, observed during the period May 1992 through November 1993. In Table 1, is given a list of these events. None of the selected XBOs were associated with any visible chromospheric activity, as seen on $\mathrm{H} \alpha$ fitergrams. In Figure 3 is shown the location of 11 events of XBOs, marked as hached area and coronal hole boundary by solid line, provided by the Space Enviornmental Services Center, NOAA, Boulder. For 4 XBO events, no coronal hole data were available. We found that in all case, XBOs were located near or at the boundary of coronal holes. Further, we compared the YOHKOH SXT pictures with low resolution Stanford longitudinal magnetic maps, available from the Solar Geophysical Data. Through precise superimposition of YOHKOH SXT data, Stanford magnetic and coronal hole maps and the $\mathrm{H} \alpha$ full disk filtergrams, it is confirmed that all non-active region XBOs near or at the coronal hole boundary, always begin to develop over the magnetic neutral line. In Figure 4 is shown a typical case of XBO (negative picture) observed on 26 January 1993 and the Stanford magnetic field map, on the right. It will be noticed that XBO is directly located over the neutral line, at the boundary of the coronal hole. We have examined all the 15 cases of $\mathrm{XBO}$, and in all cases we find that $X B O$ occur at or along the boundary of coronal holes and over the magnetic neutral line. This finding could be 
'coincidental' or 'consequential'. We believe that it is the latter, as shown in the discussion.

\section{TABLE I}

List of X-ray Blow-outs near Coronal Holes.

\begin{tabular}{|c|c|c|c|}
\hline S.No. & Date & Region & Remarks \\
\hline 1. & 27 May 92 & S-polar & No $\mathrm{H} \alpha$ feature seen near region \\
\hline 2. & $20 \mathrm{Jul} 92$ & $\mathrm{~N}-\mathrm{E}$ & No $\mathrm{H} \alpha$ feature seen near region \\
\hline 3. & 30 jul 92 & $\mathrm{~N}-\mathrm{E}$ & No $\mathrm{H} \alpha$ feature seen near region \\
\hline 4. & 18 Aug 92 & $\mathrm{~N}-\mathrm{W}$ & $\mathrm{H} \alpha$ filament seen near region \\
\hline 5. & 28 Nov 92 & $\mathrm{~N}-\mathrm{E}$ & Small $\mathrm{H} \alpha$ polar filament seen \\
\hline 6. & 14 Dec 92 & S-W & No $\mathrm{H} \alpha$ feature seen near region \\
\hline 7. & 25 Dec 92 & $\mathrm{~S}-\mathrm{W}$ & $\mathrm{H} \alpha$ filament near region seen \\
\hline 8. & 26 Jan 93 & S-W & No $\mathrm{H} \alpha$ feature seen near region \\
\hline 9. & 25 Feb 93 & S-W & $\mathrm{H} \alpha$ filament seen near region \\
\hline 10. & 10 Mar 93 & S-E & No $\mathrm{H} \alpha$ feature seen near region \\
\hline 11. & 21 Mar 93 & S-W & H $\alpha$ filament seen near C.H. \\
\hline 12. & 10 Sep 93 & $\mathrm{~N}-\mathrm{E}$ & Small $H \alpha$ filament seen \\
\hline 13. & 22 Sep 93 & S-E & No $\mathrm{H} \alpha$ feature seen near region \\
\hline 14. & 25 Oct 93 & N-E & No $\mathrm{H} \alpha$ feature seen near region \\
\hline 15. & 14 Nov 93 & N-E & No $H \alpha$ feature seen near region \\
\hline
\end{tabular}

\section{Discussion}

As all the 15 observed ' $\mathrm{X}$-ray Blow-outs' (XBOs) occured at the boundary of coronal holes and over the neutral line, it is proposed that these XBOs, which appear as enhanced soft X-ray emission measure, are generated due to the reconnection of open field lines from the coronal hole with the adjoining opposite evolving magnetic field structure. The coronal hole provides a readily available open field structure to the rising and expanding $\mathrm{X}$-ray loops or streamers. The reconnection point or region in this case would be very high in the solar atmosphere, thus no signature of any feature may be visible in the chromosphere. In Figure 5 is given a possible scenario of the proposed magnetic field configuration for generation of XBOs near coronal holes.

It is likely that these XBOs may give rise to CMEs and IPDs of varying energies. As previously pointed out by Wagner (1984) that more than $30-48 \%$ of CMEs are unrelated to any observed phenomenon on the solar surface. We suggest that at least some of these XBOs may be responsible for 

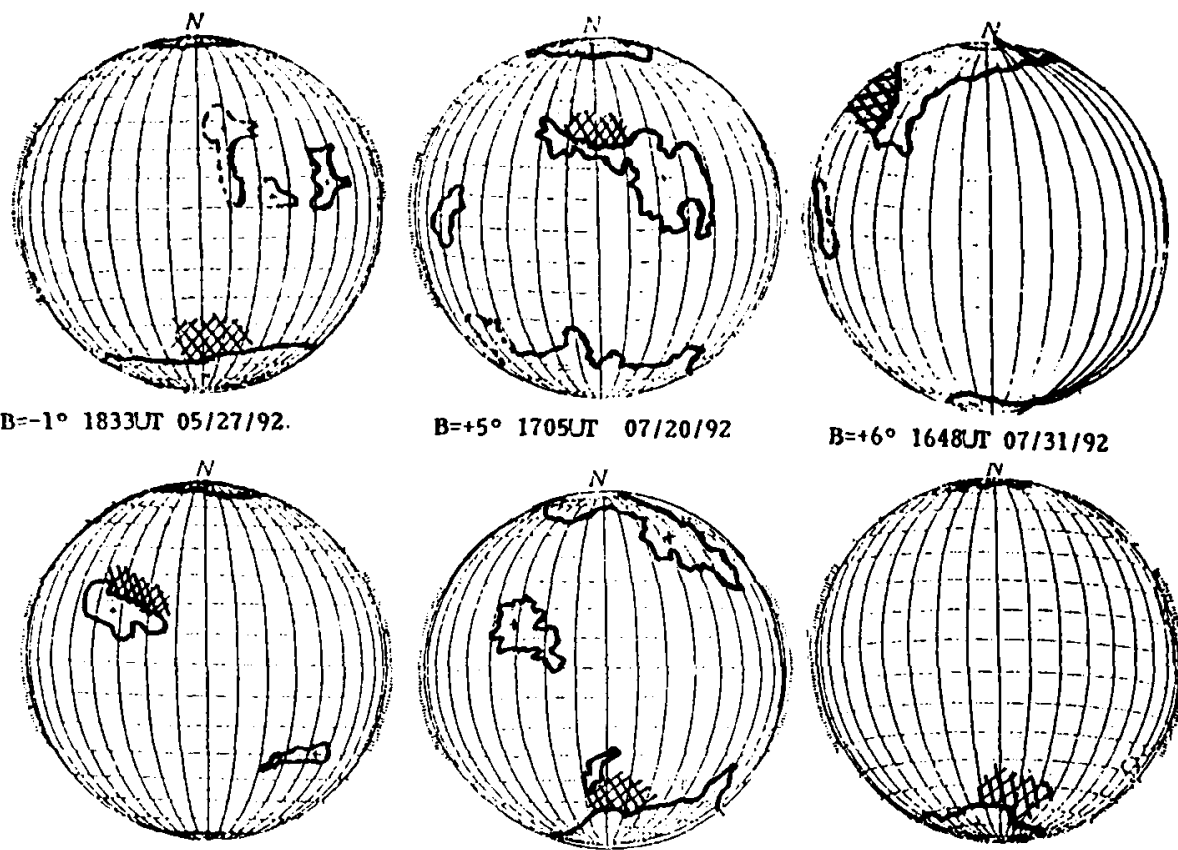

$B=+5^{\circ} \quad 1705$ Ur $07 / 20 / 92$
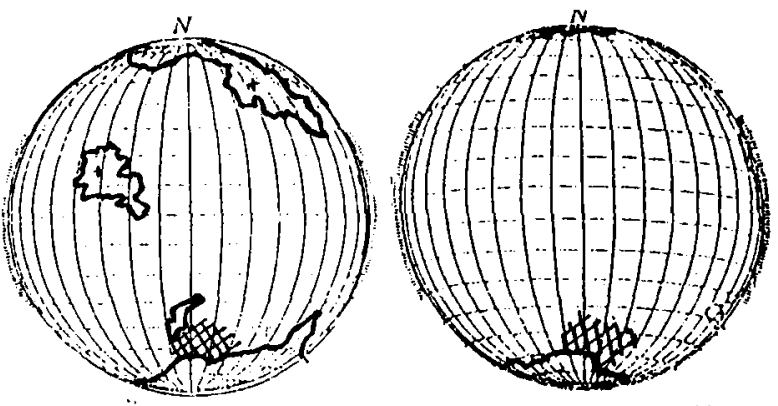

$B=+1^{\circ} 171$ Xr $11 / 28 / 92$

$\mathrm{B}=-1 \circ 1758 \mathrm{UT} 12 / 14 / 92$
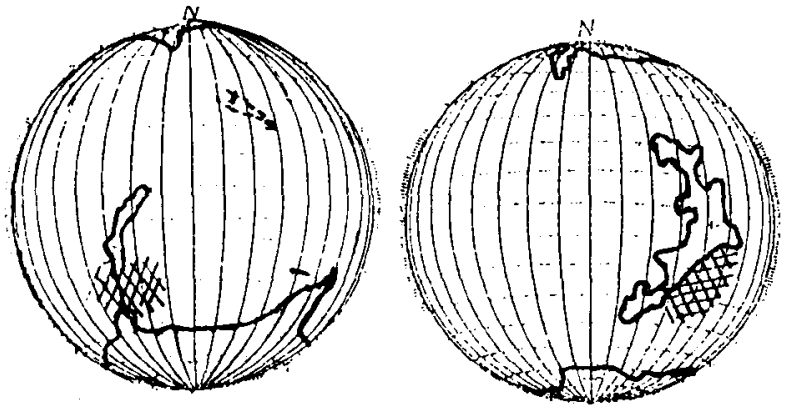

$B=-7^{\circ} \quad 2034 \mathrm{UT} \quad 02 / 25 / 93$

B--7० $1724 U \mathrm{~T} \quad 03 / 10 / 93$

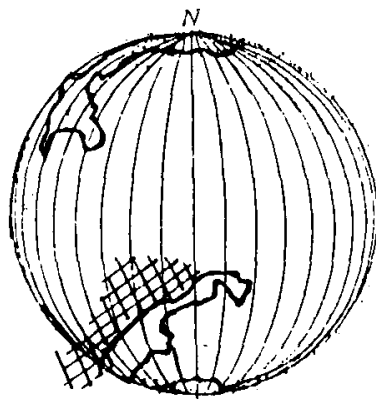

$B=-7^{\circ} \quad 155 \pi$ Tr 03/21/93
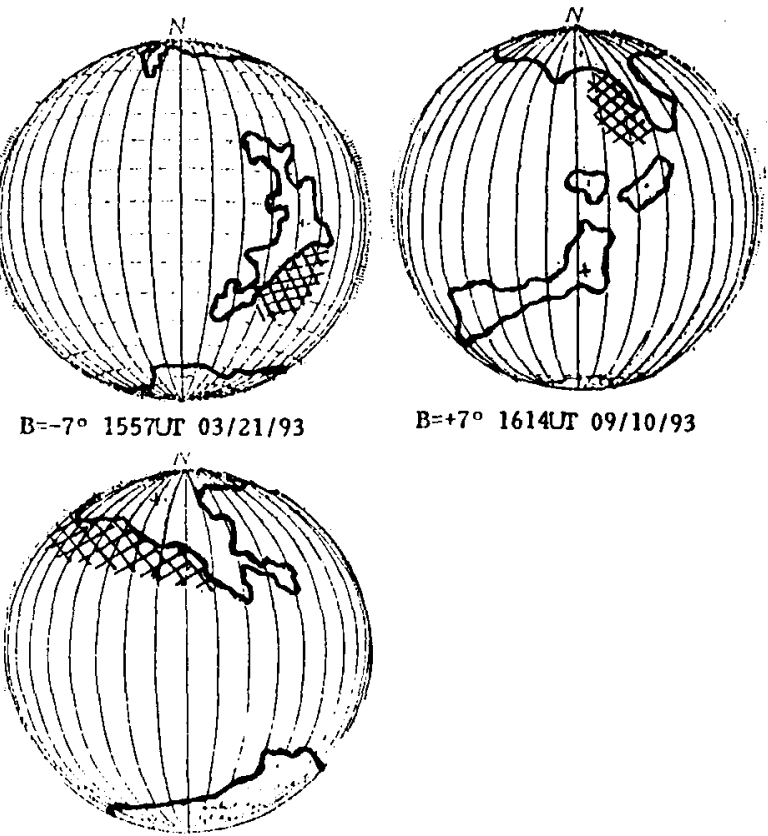

$\mathrm{B}=+7^{\circ} \quad 1614 \mathrm{UT} \quad 09 / 10 / 93$

$\mathrm{E} \cdot 77^{\circ} 2305 \mathrm{UT} 09 / 22193$

$B=+5^{\circ}$ 19132T $10 / 25 / 93$

Fig. 3. Location of coronal holes with respect to X-ray Blow-outs (XBO) 


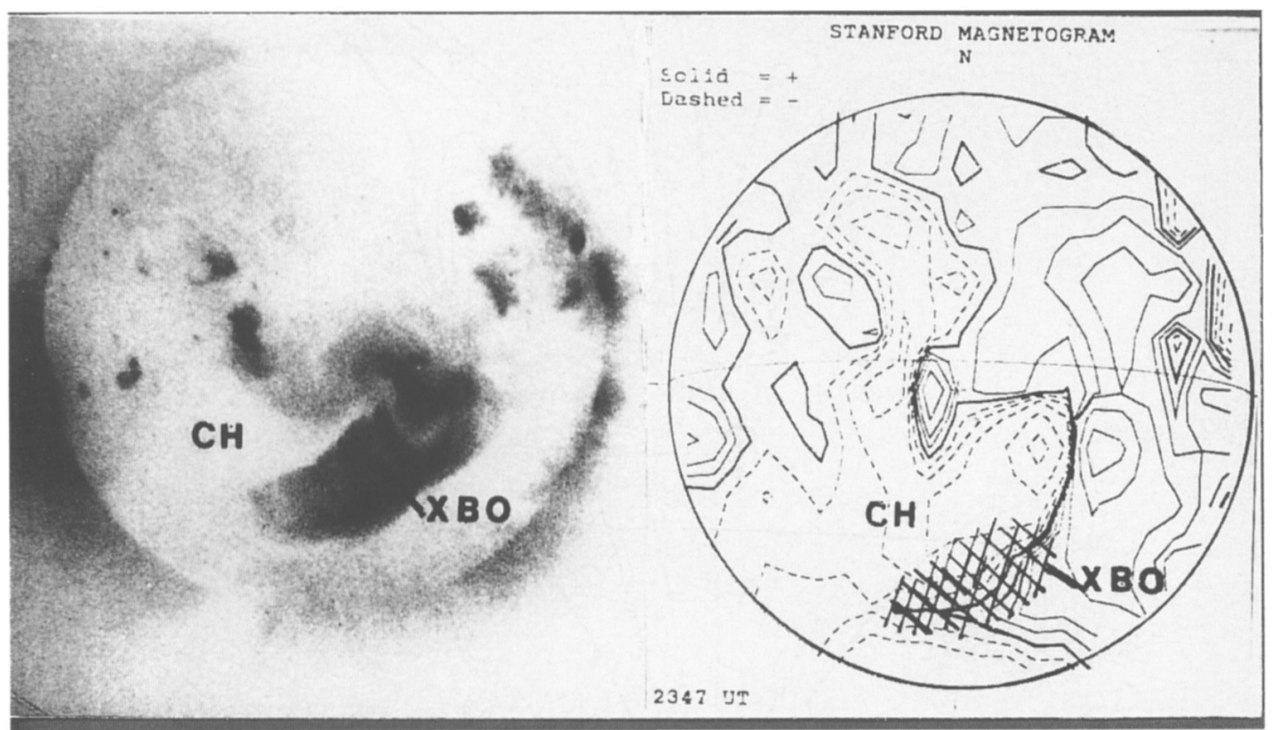

Fig. 4. YOHKOH SXT picture showing XBO observed on $26 \mathrm{Jan} .1993$ and on the right is shown the Stanford low resolution magnetic map of the same day. Note the XBO occured just over the neutral line and at the boundary of coronal hole.

the Wagner's 'unrelated' CMEs, because no surface activity is observed in the optical wavelengths, as the action takes place at much greater height in the corona. The necessary energy required for interplanetary shocks (IPS) is made available from the coronal holes, as Steinitz and Eyni (1980) have suggested that stable coronal holes are the source for excess kinetic energy flux in the solar wind. In the case of XBOs, the rising and expanding magnetic field structures provide the necessary reconnection mechanism with the near by open field lines of the coronal hole. The hot soft X-ray material then ejects out from greater heights and posssibily generate interplanetary disturbances.

\section{Acknowledgements}

The author wish to thank the Japanese Society for Promotion of Science (JSPS), the Department of Science \& Technology, Government of India and Prof. Takeo Kosugi of NAOJ, Japan for providing a visiting fellowship to study the YOHKOH data. Thanks are also due to Drs. A.Ambastha and S.C.Tripathy for helping in reducing some of the data. 


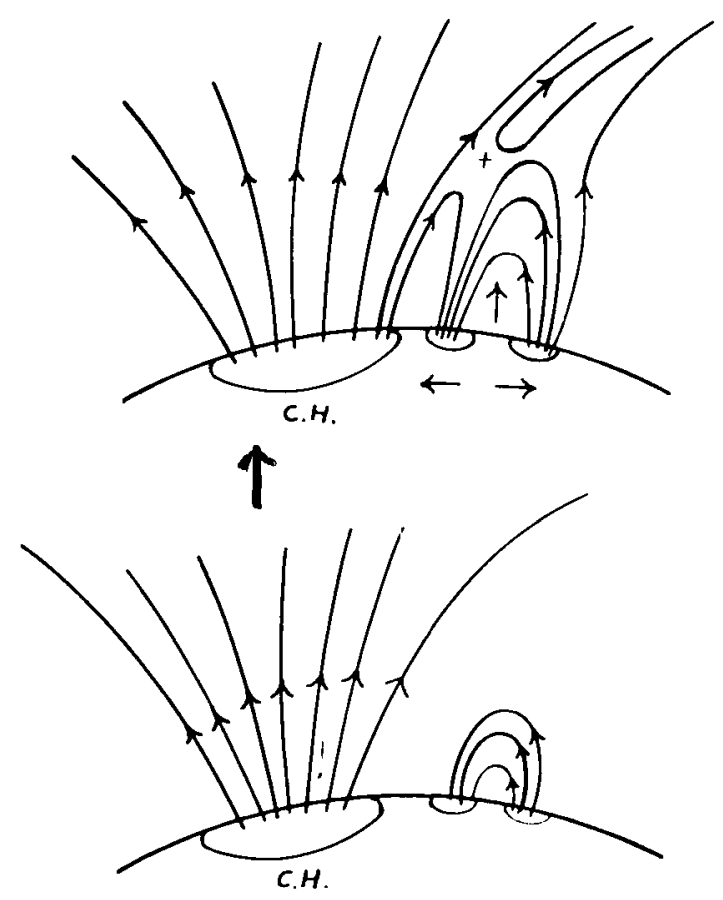

Fig. 5. A proposed scenario of magnetic field configuration resposible for XBO due to reconnection of field lines, near the boundary of coronal hole.

\section{References}

Borrini,G., Gosling,J.T., Barnes, S.J., and Feldman,W.C.:1982, J.Geophys. Res., 87,4365. Gosling,J.T.: 1993, J.Geophys. Res., 98, No.A 11, 18937.

Janardhan,P., Balasubramanian,V., Ananthakrishanan,S., Dryer,M., Bhatnagr.A., and McIntosh,P.S.: 1994 (submitted to Solar Phys.)

Joselyn,J.A., and McIntosh,R.S.: 1981, J.Geophys.Res., 86, 4555.

Kahler,S.: 1992, Ann. Revs. Astrophys., 30, 113.

Khan,J.I., Uchida,Y., McAllister,A.H., and Watanabe,Ta.: 1994 (in press)

Klimchuk,J., and 6 other authors: 1994 (in press)

Hewish,A., Tappin,S.J.,and Gapper,G.R.: 1985, Nature, 314, 137.

Hiei,E., Hundhansen,A., and Simi,D.:1993, Geophys. Res. Lett. 20. 2785.

Hudson,H.S.:1994, Proc. of Kofu Symp.

McAllister,A., and 8 other authors: 1992, Publ. Astro. Soc. Japan, 44, L205.

Sheeley,N.R.Jr., Howard,R.A., Koomen,M.J., Michels,D.J., Schwenn,R., Mulhaser,K.H., and Rosenbauer,H.: 1984, J. Geophys. Res., 90, 163.

Tsuneta,S., and 5 other authors: 1992, Publ. Astro. Soc. Japan, 44, L299.

Wagner,W.J.: 1984, Ann. Rev. Astro. Astrophys., 22, 267.

Watanabe Ta and 10 other authors.: Publ. Astro. Soc. Japan. 44, L199. 